\title{
Lexicographie et enseignement de langues liées à l'immigration: note sur le contexte sociolinguistique de l'élaboration du dictionnaire finnois-lingala-français*
}

Kadima Batumona Adi Gilbert, Centre de recherche pour l'instrumentalisation du cilubà (CRIC / Finlande), Centre de linguistique théorique et appliquée (CELTA / RD-Congo), Ghent University, Ghent, Belgium (adi_kadima@ymail.com)

Les étrangers, y compris les migrants ont le droit de conserver leur langue maternelle, leur culture et leurs traditions.

Résumé: Le dictionnaire finnois-lingala-français dont il est question dans cette étude a été rédigé dans un contexte d'immigration. Il est l'illustration-même du soutien apporté aux migrants par leurs pays d'accueil en vertu du droit à la langue reconnu à tout être humain et au nom du droit international instituant les droits culturels des migrants. Notre contribution à cette thématique tout en se voulant linguistique, aborde quelques aspects de ces droits culturels des migrants en ce que leur reconnaissance a occasionné une activité lexicographique portant sur le lingala, langue dont la présence en Finlande ne se justifie que par l'immigration.

Mots-clés: LEXICOGRAPHIE, ENSEIGNEMENT DE LA LANGUE MATERNELLE, IMMIGRATION, DROITS DE L'HOMME, DROITS CULTURELS DES MIGRANTS, PLURILINGUISME, DROIT À LA LANGUE

Abstract: Lexicography and Language Teaching Related to Immigration: Note on the Sociolinguistic Context of the Development of the FinnishLingala-French Dictionary. The Finnish-Lingala-French Dictionary discussed in this article was compiled within the context of immigration. It illustrates the support for migrants in their host country under the right to language recognized for every human being and under international law establishing the cultural rights of migrants. Our contribution to this issue, intended to be linguistic, addresses some aspects of the cultural rights of migrants whereby their recognition has resulted in a lexicographical activity concerning Lingala whose presence in Finland can only be explained because of immigration.

Texte remanié et mis à jour de la communication intitulée Les problèmes posés par l'élaboration du dictionnaire finnois-lingala-français, présentée au XVIIème Congrès des Romanistes Scandinaves, Université de Tampere, Finlande, 12-15 août 2008. 
Keywords: LEXICOGRAPHY, LANGUAGE TEACHING, IMMIGRATION, HUMAN RIGHTS, CULTURAL RIGHTS OF MIGRANTS, MULTILINGUALISM, RIGHT TO LANGUAGE

\section{Introduction}

\subsection{Objet de l'étude et objectif visé}

L'intitulé du présent article nécessite quelque précision ou délimitation, car il laisse penser que l'objet de notre contribution consisterait en la description de la manière dont notre dictionnaire est utilisé en Finlande dans l'enseignement. Ce qui est une des possibilités, à première vue. Mais en nous basant sur la formulation de son sous-titre, son objet se laisse également saisir comme consistant en la description d'une part, du contexte sociolinguistique dans lequel ce dictionnaire finnois-lingala-français, destiné à être utilisé comme outil d'appui à l'apprentissage a vu le jour et d'autre part, à démontrer que l'immigration en tant que phénomène social, est à même de générer une activité lexicographique ou une dictionnairie.

En effet, ce dictionnaire qui a vu le jour dans ce particulier contexte d'immigration, nous sert d'exemple-type du soutien apporté aux migrants par les municipalités de leurs pays d'accueil et cela, au nom du droit international recommandant la reconnaissance ainsi que le respect de leurs droits culturels.

Mais notre contribution à cette dernière thématique demeure donc essentiellement linguistique.

\subsection{Migration et langues liées à l'immigration (LLI)}

La disposition de l'article 5.1.f de la Déclaration sur les droits de l'homme des personnes qui ne possèdent pas la nationalité du pays dans lequel elles vivent couvre juridiquement les migrants et résume à elle seule l'essentiel de leurs droits culturels. C'est au nom de ces droits culturels que dans beaucoup de pays européens les migrants se voient accordés certains droits spécifiques liés à leurs origines ainsi qu'à leurs langues et soutenus dans la pratique ainsi que dans l'enseignement de langues venant de leurs pays d'origine (ONU 1984).

D'ordinaire, les personnes qui émigrent à l'étranger prennent avec elles souvent quelques-uns de leurs effets personnels jugés importants, c'est-à-dire des bagages de première nécessité. Ces personnes soupçonnent rarement que tandis qu'elles émigrent, elles emportent également avec elles d'autres bagages plus importants: leurs langues maternelles, véhicules de leurs cultures et port d'attache à leurs origines traditionnelles!

Ces langues, étant donné qu'en Afrique, de nombreux pouvoirs publics ne les aménagent le plus souvent pas en vue de leur instrumentalisation, entretien ou équipement, doivent leur survie seulement grâce au fait qu'elles servent de moyen de communication au sein d'une même communauté linguistique ou entre les personnes issues de communautés linguistiques différentes. C'est par- 
tant de cette situation de délaissement total que ces langues se retrouvent aujourd'hui parmi celles que les spécialistes de politique linguistique s'accordent à qualifier de langues peu dotées, ce qui veut dire qu'elles figurent parmi celles des moins outillées au monde.

Mais, une fois déballées sur le vieux continent, en vertu du principe de respect aux droits culturels des migrants ou celui du droit à la langue maternelle, ces langues délaissées se voient soudainement protégées et acquièrent des statuts qu'elles n'ont pas dans leurs milieux naturels, retiennent l'attention des pouvoirs publics des pays ayant accueilli leurs locuteurs et deviennent des matières potentielles d'enseignement pour les enfants des migrants. C'est dans ce contexte que diverses terminologies ont vu le jour en Europe et plus particulièrement dans les pays nordiques scandinaves pour désigner et différencier ces langues de langues européennes: langues liées à l'immigration, langues d'origine, langues d'Europe, etc., terminologies que nous définirons plus loin.

\subsection{Les LLI face à complexité linguistique de l'Europe}

Nombreux parmi nous sont des personnes d'origine immigrée qui se souviennent de cette phrase: «L'Europe ne peut pas accueillir toutes les misères du monde», devenue depuis lors des plus célèbres et dont l'auteur n'est plus à présenter. Cette phrase revient le plus souvent dans les discours de certains leaders politiques dont nombreux se recrutent de l'extrême-droite et est utilisée aux fins de traduire l'hostilité ou la xénophobie que manifestent sur le territoire européen ces leaders face aux entrées effrénées, parfois massives et souvent illégales des populations étrangères.

Parallèlement à ces discours hostiles ou xénophobes, l'Europe ou mieux l'Union européenne, pour sa part, se montre humaniste, ouverte et prône une immigration qui respecte les droits des hommes et des États en même temps qu'elle s'interroge sur son identité, sur les possibilités d'en définir le contour sans pour autant pratiquer l'exclusion des populations qui viennent de tous les coins du monde pour y vivre ${ }^{1}$. Ce qui va sans dire que l'Europe, en tant que terre d'immigration, est un espace où se parlent plusieurs langues, celles du terroir et celles liées à l'immigration, c'est-à-dire toutes celles que les migrants portent avec eux à leur installation sur le territoire européen. D'où la diversité linguistique de l'Europe qui compte dès lors non seulement ses propres langues (dites langues européennes) mais également toutes celles portées par les migrants (dites langues liées à l'immigration).

\subsection{Quelques définitions utiles}

\section{- Les langues européennes}

Dans les discours relatifs à la «diversité linguistique de l'Europe», les «langues européennes» sous-entendent uniquement les langues des natifs d'origine 
européenne et comprennent les langues indo-européennes (entre autres les langues romanes), les langues finno-ougriennes et les langues sémitiques.

\section{- Les langues d'Europe}

Elles désignent aussi bien les langues européennes que les langues non-européennes (langues des minorités immigrées et réfugiées) en usage sur le territoire européen (comme c'est le cas du lingala, langue afro-congolaise et qui est la principale langue cible du dictionnaire dont il est question dans la présente contribution.

\section{- Les langues liées à l'immigration (LLI)}

L'emploi par Quentin (2002) de cette terminologie langues liées à l'immigration doit être perçu comme un relais du discours politique relatif à l'immigration. La réalité que couvre cette terminologie de «LLI» est diversement dénommée aussi bien dans la littérature scientifique que dans le vocabulaire politique européen relatif à l'immigration.

Aux côtés de cette terminologie de «LLI», nous en avons inventorié d'autres, à savoir: langues de l'immigration (Unesco 2003), langues dites d'immigration (Bouquin-Keller 2001) et langues des enfants issus de l'immigration (Huck 2012). Qu'il s'agisse de l'une ou de l'autre terminologie parmi celles que nous venons d'énumérer, nous nous trouvons dans tous les cas face aux dénominations nonconsacrées et qui sont absentes du Cadre européen commun de référence aux langues (CECRL). La compréhension que nous faisons de ces terminologies est qu'elles se réfèrent toutes aux idiomes que les personnes immigrées ou toutes celles en instance d'immigration ont comme langues maternelles ou premières langues.

Toutefois, nous avons opté pour l'emploi de la terminologie langues liées à l'immigration, car elle traduit mieux cette jonction qu'il y a entre langue et immigration. C'est de cette jonction langue et immigration que découle la lexicographie de langues liées à l'immigration, notre centre d'intérêt dans le cadre de la présente description.

\section{La lexicographie de langues liées à l'immigration (LLLI)}

L'institution de cette terminologie de «langues liées à l'immigration», l'on s'en doute, ne peut rester sans conséquence pour d'autres branches du savoir humain qui sont liées à l'instrumentalisation ou mieux à l'équipement des langues. C'est dans ce contexte, par exemple, que la l'élaboration d'un dictionnaire comme celui portant sur le finnois, le lingala et le français, étant donnée les raisons justifiant la présence du lingala sur le territoire européen (entre autre en Finlande), convient d'être inscrite dans le cadre de ce que nous avons, par extrapolation, jugée bon de dénommer «la lexicographie de langues liées à l'immigration». 


\subsection{Quelques précisions terminologiques}

Rappelons que selon le CNRTL ou le Centre national de ressources textuelles et littéraires (service spécialisé du CNRS), la lexicographie est la technique de confection des dictionnaires et l'étude scientifique et analytique des faits de lexique. C'est une discipline linguistique dont l'objet est la connaissance, l'étude des mots et des expressions d'une langue déterminée, et qui vise en particulier, à l'élaboration des dictionnaires (Larousse 2009).

La lexicographie de langues liées à l'immigration ou «LLLI», selon nous, pourrait s'entendre comme l'ensemble de pratiques dictionnairiques qui visent l'équipement en outils lexicographiques des langues liées à l'immigration. Cette dénomination «LLLI» est de toute apparence nouvelle, mais lorsque nous examinons l'objet ainsi que les objectifs de la sous-discipline désignée, nous nous rendons vite à l'évidence que cette dernière a des rapports étroits avec la lexicographie d'apprentissage (LA) ou la lexicographie pédagogique (LP), dénominations des plus connues en sociolinguistique, en didactique ou en lexicographie.

\subsection{Lien entre la LLLI et la LP ou la LA}

Tout en tenant pour acquis les précisions terminologiques que nous venons de faire quant à la LLLI, examinons à présent le lien qui existerait entre elle (la LLLI) et ce que l'on nomme LA ou LP.

En effet, le GRELEP (Groupe de recherche en lexicographie pédagogique) décrit la lexicographie pédagogique ou lexicographie d'apprentissage comme une sous-discipline de la lexicographie qui se propose d'élaborer des dictionnaires (en papier ou électroniques) dont l'objectif est de répondre aux besoins des apprenants d'une langue, tant du point de vue réceptif (compréhension du message) que du point de vue productif (mise en discours d'un message).

Rappelons-le et ce, comme nous l'avons déjà mentionné ci-haut que notre «LLLI» se propose d'équiper une «LLI» en outils lexicographiques pour les besoins de ses locuteurs en leur conférant par le truchement de l'usage du dictionnaire la maîtrise des moyens d'expression ainsi que l'accroissement du savoir culturel. Comme nous pouvons bien nous en rendre compte, entre ce que nous désignons par la «LLLI» et ce que le GRELEP nomme la LP ou LA, la différence n'est que dénominationnelle, car les objets d'étude ainsi que les buts visés par les deux sous-disciplines lexicographiques demeurent identiques. Mais la particularité de la LLLI tient à la jonction entre langue et immigration dont elle est le produit ou le résultat. Bien plus, la LLLI est de par son objectif une LP ou LA tandis que toute LP ou LA n'est pas forcément une LLLI. 


\subsection{LLLI et LLI en Finlande: état de lieu}

\subsubsection{La lexicographie de langues liées à l'immigration}

En Finlande, les dictionnaires sont élaborés et utilisés depuis plusieurs centaines d'années et interviennent dans toutes les formes d'interaction entre les locuteurs de langues différentes. Ils sont également utilisés comme matériels d'appui destinés aussi bien à la recherche qu'à l'enseignement ou l'apprentissage des langues maternelles et étrangères. C'est ce qu'il faut entendre par cette phrase en finnois de Romppanen (2001):

«Sanakirjoja on Suomessakin laadittu ja käytetty monen sadan vuoden ajan apuneuvoina koulutuksessa, tutkimuksessa ja kaikenlaisessa kanssakäymisessä erikielisten kesken».

Pour ce qui est du nombre des dictionnaires existants, on en dénombre près d'un millier. Il suffit de se rendre sur www.helmet.fi et d'interroger la base à l'aide des mots «sanakirja» (en finnois) ou «dictionary» (en anglais) pour se rendre vite à l'évidence que pour la seule région de la capitale Helsinki, il y a environ 1778 dictionnaires en circulation. Le tableau répertoriant pour l'année 2011 les langues étrangères enseignées en Finlande (cf. section 2.3.2.) mentionne aux côtés de chacune d'entre elles, les dictionnaires disponibles. L'absence de tout chiffre veut dire qu'il n'existe pas de dictionnaire pour la langue étrangère concernée. Ce que nous pouvons dire quant à l'état de lieu de LLLI en Finlande est que sur les 147 LLI parlées en Finlande, 53 langues ont été enseignées en 2011, 28 LLI parmi ces 53 suscitées disposent des outils lexicographiques de référence ou d'appui à l'apprentissage.

\subsubsection{L'enseignement des LLI en Finlande}

On peut lister plusieurs activités qui entretiennent des rapports étroits avec la lexicographie. Pour notre part, dans le cadre de la présente contribution, nous en avons identifié un, à savoir l'enseignement et/ou l'apprentissage de la langue maternelle, pour lequel les dictionnaires demeurent des ouvrages fondamentaux. En Finlande, cet enseignement n'est pas obligatoire, mais pour pouvoir l'organiser, deux conditions sont requises, à savoir: avoir au minimum une classe de 4 apprenants et un enseignant ayant reçu, de l'Office finlandais en charge de l'enseignement, l'habilitation à dispenser cet enseignement.

Le tableau ci-dessous fait état des cours de langue maternelle, langue de la maison ou langue d'entretien qui ont été organisés à travers la Finlande au cours de l'année 2011. Ces langues étant presque toutes étrangères à la Finlande (excepté le saami et le suédois), nous les considérons comme des LLI. Celles suivies d'un astérisque sont d'origine africaine et comme les chiffres l'indiquent, le lingala figure parmi les rares LLI d'origine africaine enseignées en Finlande. L'on notera en passant que le somalien figure parmi les grandes LLI 
en Finlande, car il compte parmi celles qui ont un nombre plus élevé d'apprenants (2197 au total pour la seule année 2011) (cf. Opetushallitus s.d).

\begin{tabular}{|c|c|c|c|c|c|c|}
\hline $\begin{array}{c}\text { Langue } \\
\text { enseignée }\end{array}$ & Apprenants & \multicolumn{2}{|c|}{$\begin{array}{l}\text { Dictionnaires } \\
\text { disponibles }\end{array}$} & $\begin{array}{c}\text { Langue } \\
\text { enseignée }\end{array}$ & Apprenants & $\begin{array}{l}\text { Dictionnaires } \\
\text { disponibles }\end{array}$ \\
\hline albanais & 775 & & & lingala* & 20 & 1 \\
\hline allemand & 192 & \multicolumn{2}{|c|}{40} & lituanien & 4 & 4 \\
\hline amharique* $^{*}$ & 14 & & & népalais & 44 & \\
\hline anglais & 386 & \multicolumn{2}{|c|}{97} & norvégien & 27 & 4 \\
\hline arabe & 1098 & \multicolumn{2}{|c|}{7} & ourdou & 77 & \\
\hline azéri & 6 & \multicolumn{2}{|c|}{7} & pashtou & 22 & \\
\hline bengali & 68 & & philippin & 53 & \\
\hline birman & 103 & & & polonais & 73 & 3 \\
\hline bosnien & 127 & \multicolumn{2}{|c|}{1} & portugais & 33 & 4 \\
\hline chinois & 334 & \multicolumn{2}{|c|}{3} & roman & 164 & \\
\hline dari & 169 & & & roumain & 18 & 2 \\
\hline espagnol & 262 & \multicolumn{2}{|c|}{17} & russe & 3770 & 56 \\
\hline estonien & 740 & \multicolumn{2}{|c|}{16} & sami & 44 & \\
\hline farse & 351 & \multicolumn{2}{|c|}{1} & serbe & 5 & \\
\hline français & 164 & \multicolumn{2}{|c|}{37} & somali* & 2197 & 1 \\
\hline grèc & 63 & \multicolumn{2}{|c|}{1} & suédois & 17 & 65 \\
\hline hébreu & 28 & \multicolumn{2}{|c|}{1} & tamoul & 14 & \\
\hline hindi & 24 & & & tchèque & 7 & \\
\hline hollandais & 18 & \multicolumn{2}{|c|}{4} & tchétchène & 25 & \\
\hline hongrois & 85 & & & thaï & 285 & 1 \\
\hline islandais & 11 & & tigrinya & 20 & \\
\hline italien & 104 & \multicolumn{2}{|c|}{12} & turc & 173 & 1 \\
\hline japonais & 46 & \multicolumn{2}{|c|}{1} & turkmène & 7 & \\
\hline khmer & 9 & & & uighur & 7 & \\
\hline kirundi* & 13 & & & ukrainien & 8 & 1 \\
\hline kurde & 454 & \multirow{2}{*}{\multicolumn{2}{|c|}{4}} & vietnamien & 476 & 1 \\
\hline \multirow[t]{4}{*}{ letton } & 10 & & & & & \\
\hline & \multicolumn{5}{|c|}{ 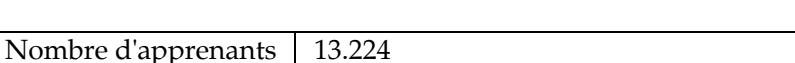 } & \\
\hline & \multicolumn{2}{|c|}{ Langues enseignées } & \multicolumn{3}{|c|}{$\begin{array}{l}53 \text { dont } 28 \text { équipées de plus au moins } \\
\text { un dictionnaire }\end{array}$} & \\
\hline & \multicolumn{2}{|l|}{ Organisateurs } & \multicolumn{3}{|c|}{92} & \\
\hline
\end{tabular}

Les LLI en Finlande étant pour la plupart des langues étrangères, leur enseignement ou apprentissage a lieu au cours de 10 premières années que dure l'enseignement fondamental. Pour ce qui en est des bénéficiaires, l'on notera que cet enseignement ou apprentissage est destiné aux enfants appartenant à l'une ou l'autre catégorie parmi celles ci-après: enfants bilingues ayant pour langues maternelles autres langues que le finnois ou le suédois; enfants saamephones; enfants issus de familles d'origine tsigane; enfants finlandais ayant vécu à l'étranger et ceux ayant été scolarisés en langues étrangères tandis qu'ils séjournaient hors de la Finlande (cf. Helsingin Kaupunki-Opetusvirasto s.d.). 


\subsection{La Finlande et la gestion de son plurilinguisme}

Il est acquis en droit international qu'aucun État n'est obligé d'accueillir un non ressortissant sur son territoire. En revanche, lorsque ce non ressortissant, migrant ou étranger remplit les conditions de son admission sur le territoire, l'État concerné ne peut plus lui refuser la jouissance des droits garantis par les traités internationaux de droits de l'homme (cf. Agbetse s.d.). À titre d'exemple, parmi ces traités internationaux, nous avons mentionné en introduisant cette contribution, la Déclaration sur les Droits de l'homme des personnes qui ne possèdent pas la nationalité du pays dans lequel elles vivent dont nous avons épinglé l'article 5.1.f qui reconnaît aux personnes d'origine immigrée le droit de conserver leur langue maternelle, leur culture ainsi que leurs traditions.

Cette reconnaissance de jouissance des droits culturels des migrants, garantis par le droit international implique à son tour en Finlande la reconnaissance du droit à l'instruction pour lequel les municipalités se voient obligées de satisfaire aux exigences du programme d'enseignement en instaurant un soutien spécifique de langue d'origine aux enfants d'origine immigrée et qui n'ont ni le finnois ni le suédois pour langues maternelles.

En contexte de crise, garantir ce droit à la langue s'avère coûteux pour un pays d'accueil comme la Finlande qui se verrait obligée d'organiser par exemple les enseignements de 147 langues maternelles parlées sur son territoire, acheter le matériel didactique destiné à cet enseignement, financer l'élaboration des outils lexicographiques si faire se peut, payer les salaires aux enseignants de ces langues, etc. Ne disposant pas des moyens financiers suffisants, le gouvernement finlandais a planifié les choses comme bon lui semble en faisant la sourde oreille aux plaintes des mécontents: ainsi par exemple, tous les ressortissants congolais qui ont comme langues maternelles le ciluba, le kiswahili, le kikongo, etc. se voient obligés d'apprendre le lingala!

\subsubsection{Statut des LLI en Finlande}

Quel que soit le privilège dont les langues liées à l'immigration jouissent en vertu des droits spécifiques accordés ou reconnus à leurs locuteurs, du fait de leurs origines étrangères et ce, au nom du droit international ou simplement du droit à la langue, ces langues restent des langues étrangères.

Pour ce qui concerne aussi bien l'usage que l'apprentissage de ces langues, il importe de le souligner ainsi que le note l'Unesco que les États européens (surtout ceux du Sud et de l'Ouest) n'ont pas pris de dispositions particulières pour l'enseignement et la pratique des langues de l'immigration (entendez les LLI selon la terminologie que nous utilisons). La raison justifiant cette absence est que certains décideurs politiques européens dans ces derniers États considèrent que l'apprentissage ou l'enseignement des LLI constitue un des obstacles à l'intégration des populations immigrées (cf. Unesco 2003).

Par contre, dans les pays européens nordiques ou scandinaves et plus par- 
ticulièrement en Finlande, il existe un programme officiel d'enseignement et d'apprentissage de toutes langues étrangères, localement dénommé «oman äidinkielen, kotikielen ja ylläpitokielen opetus» pouvant se traduire littéralement par enseignement de sa propre langue maternelle, de la langue de la maison et de la langue d'entretien. Le lingala étant non seulement une LLI, mais également une langue étrangère, son enseignement est assuré dans ce même cadre.

\subsubsection{Les LLI dans la vie socioculturelle en Finlande}

Les LLI ne sont pas seulement des matières d'enseignement ou des langues d'appui à l'enseignement en Finlande (les véhicules officiels sont le finnois et le suédois). Elles sont également des langues de travail, car elles sont présentes dans l'administration locale (hôpitaux, police, crèches, tribunaux, etc.) et sont utilisées par environ $22 \%$ de la population active.

En effet, tandis que le Centre finlandais des statistiques avait noté que pour l'année 2010, le nombre d'habitants en Finlande était de 5375276 personnes dont 248135 étaient reconnues d'origine étrangère (cf. Väestörekisterikeskus 2011: 2), l'Office finlandais au 31 décembre 2011, revoyait ce chiffre à la baisse et notait que la population immigrée résidant de manière permanente en Finlande était de 183133 personnes (cf. Sisäasiainministeriö 2011: 4).

Pour ce qui est des langues parlées par ces populations d'origine étrangère, le même Centre finlandais des statistiques avance le chiffre de 147 langues dont 43 parmi ces dernières comptent plus au moins 500 locuteurs. (cf. Väestörekisterikeskus 2011: 2). Il s'agit de:

\begin{tabular}{l|l|l|l} 
afghan & chinois & lingala & russe \\
albanais & espagnol & néerlandais & serbo-croate \\
allemand & estonien & norvégien & somali \\
amharique & français & ourdou & swahili \\
anglais & grec & pakistanais & tamoul \\
arabe & hindi & persan & télougou \\
bengali & italien & philippin & thaï \\
birman & japonais & polonais & turc \\
bosnien & kurde & portugais & ukrainien \\
bulgare & letton & roumain & vietnamien
\end{tabular}

Les diffuseurs institutionnels ainsi que les entreprises opérant dans domaine de la traduction et de l'interprétation dressent, pour leur part, la liste de ces 80 langues les plus demandées en Finlande (cf. Helsingin Tulkkikeskus Oy):

\begin{tabular}{l|l|l|l} 
akan & français & lituanien & roumain \\
albanais & géorgien & lu & russe
\end{tabular}




\begin{tabular}{l|l|l|l} 
allemand & grecque & macédoine & serbe \\
amharique & haoussa & malayalam & serbo-croate \\
anglais & hébreu & manding & slovaque \\
arabe & hindi & marathi & somali \\
arménien & hongrois & néerlandais & suédois \\
azéri & igbo & norvégien & swahili \\
bengali & indonésien & nuer & tamoul \\
berbère & ingouche & oromo & tatar \\
birman & italien & ouighour & tchèque \\
bosnien & japonais & ourdou & tchétchène \\
bulgare & jolla & ouzbek & thaï \\
chinois & kikongo & pachto & tigrinya \\
cinghalais & kikuyu & panjabi & turc \\
croate & kinyaruanda & persan & turkmène \\
dari & kirundi & peul & ukrainien \\
espagnol & krio & philippin & vietnamien \\
estonien & kurde & polonais & wolof \\
finnois & lingala & portugais & yoruba
\end{tabular}

\subsubsection{Les outils lexicographiques disponibles}

Il est quasi-universellement admis que les dictionnaires usuels, les grammaires et les atlas font partie des ouvrages de référence d'usage courant, dont la fréquence d'utilisation en classe est en général élevée et qui sont aussi considérés comme du matériel didactique de base (cf. MELS-Québec 2013).

En Finlande, le ministère de l'éducation partage cet avis et sur son site www.edu.fi, il a établi une classification des ouvrages de référence, semblable à celle en usage au Québec et dresse la liste des dictionnaires approuvés et qui peuvent être utilisés comme matériels d'appui dans l'apprentissage des LLI en usage en Finlande. Cette liste date de 2001 et n'est malheureusement pas actualisée. Le jour où elle le sera, elle reprendra certainement notre dictionnaire finnois-lingala-français, car approuvé par les spécialistes finlandais (cf. section 3.2) et il est utilisé depuis plus de six ans comme matériel didactique dans beaucoup d'établissements scolaires en Finlande, où sont organisés les cours du lingala comme langue étrangère et maternelle.

C'est à ce titre que la Bibliothèque municipale de Helsinki le répertorie dans sa banque des données parmi les quelques rares documents utilisés comme matériel didactique dans les établissements d'enseignement fondamental. Comme les lecteurs peuvent bien s'en rendre compte, c'est bien ledit dictionnaire qui vient en premier lieu à l'interrogation de la banque à l'aide du mot «lingala» comme critère de recherche. 


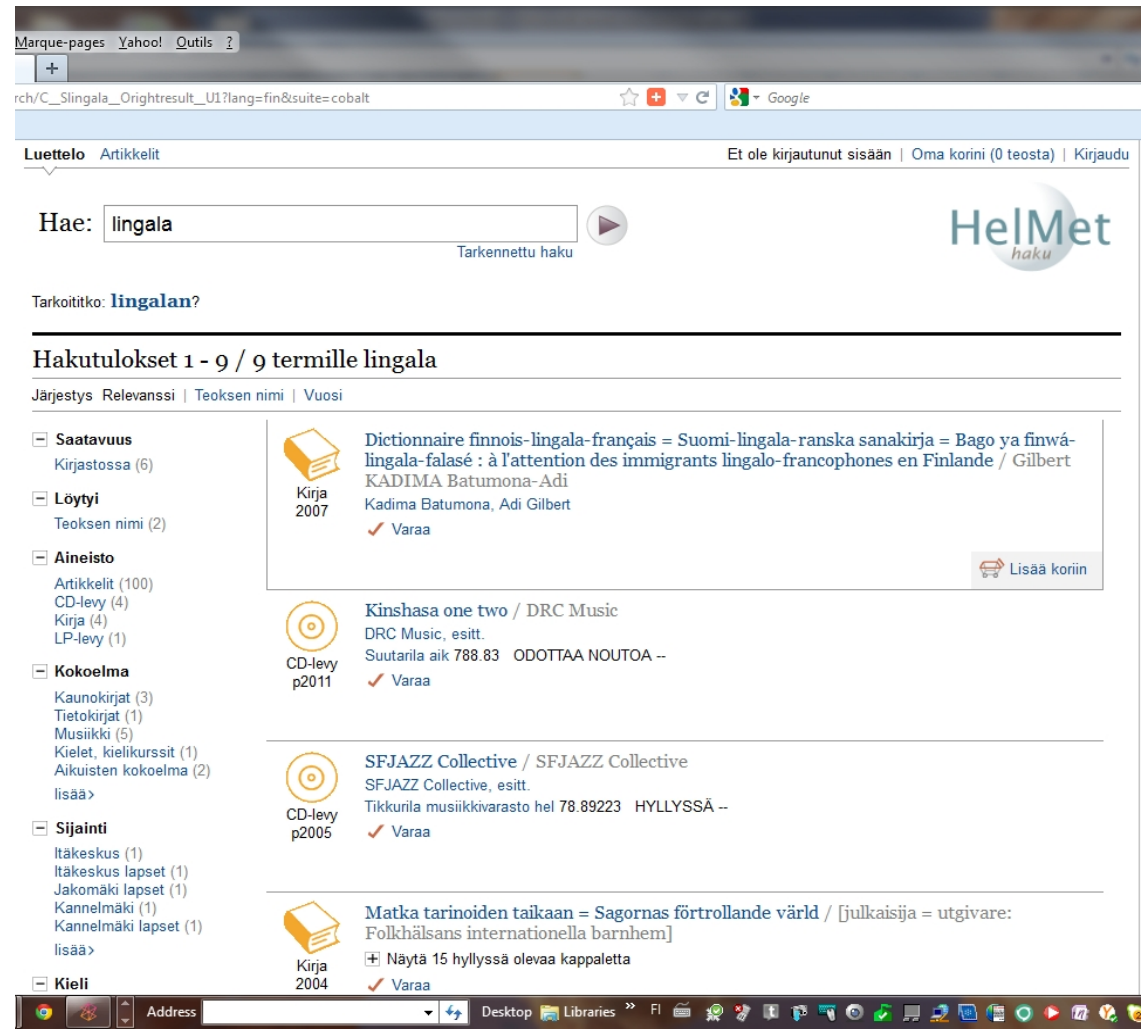

\section{Notre contribution: le dictionnaire finnois-lingala-français}

L'implantation d'une langue liée à l'immigration peut susciter, dans certaines conditions, des recherches lexicographiques à même d'aboutir à la dictionnairie. C'est ce qui nous fait dire que l'immigration est un facteur de production dictionnairique. Mais qu'est-ce qu'un dictionnaire?

Murray (s.d.) écrit à propos du mot «dictionnaire» qu'il est un livre qui traite des mots isolés d'une langue afin de montrer leur orthographe, leur prononciation, leur dérivation et leur histoire ou au moins certains de ces faits. Pour la commodité du classement, les mots sont placés dans un ordre déterminé, alphabétique dans beaucoup de langues.

\subsection{Corpus et méthodologie}

De nos jours, une description de nature linguistique ou littéraire se conçoit mal sans un échantillon d'étude ou mieux sans corpus. C'est dans ce même ordre d'idées que Cabré (2000: 14) recommande que le «linguiste qui veut décrire le langage et les langues commence toujours avec quelques données sur les langues. La provenance de ces données est le discours oral ou écrit des sujets 
parlants. Un linguiste concerné par la description des unités terminologiques doit les chercher dans les productions orales et écrites des spécialistes».

Ne pouvant pas déroger à cette exigence méthodologique, dans l'élaboration de notre dictionnaire finnois-lingala-français, nous avons pris soin de constituer un corpus dont les sources principales sont des outils didactiques, lexicographiques et terminologiques qui traitent du finnois, langue source de ce dictionnaire. Les plus importants des documents dépouillés ont été:

(a) Dictionnaire finnois-français-finnois d'Hélène Lattunen et Kari Viljanen, 2ème édition de 2000;

(b) Suomi-englanti-suomi sanakirja de Raija Hurme et al., 10ème édition de 2007; et

(c) Dictionnaire bangala-français-lingala de Atibakwa Baboya Edema, 1ère édition de 1994.

Notre dictionnaire traduit celui de Lattunen et Vijanen et c'est sur base de ce dernier que nous avons établi notre nomenclature. Nous avons recouru aux deux autres dictionnaires figurant sur la liste seulement lors de la recherche des équivalences.

Une remarque s'impose quant à l'absence parmi nos sources de référence de deux autres dictionnaires lingala des plus connus: il s'agit en effet du dictionnaire de Kawata Ashem Tem et du dictionnaire de René van Everbroeck auxquels nous n'avons pas pu accéder. Nous pensons nous corriger cette lacune avec la publication prochaine de l'édition à large diffusion en cours de préparation.

Pour ce qui est de la méthodologie nous avons eu recours à divers outils méthodologiques qui partent des observations passives mais soutenues aux dépouillement d'outils lexicographiques existant dans la langue source, le finnois.

Ces observations qui avaient porté sur le paysage linguistique de la Finlande, nous ont conduit à poser un diagnostic, à savoir: l'inexistence d'un dictionnaire finnois-lingala. De ce diagnostic, nous avions, en tant que linguiste lexicographe, réalisé qu'il y avait réellement un «besoin» à satisfaire. Le mot «besoin» n'est pas à entendre ici seulement en termes de demande, de motivation ou d'attente, mais au sens lui conféré par les spécialistes de l'analyse de besoins au Québec, celui d'«écart mesurable qui existe entre une situation actuelle («ce qui est») et une situation désirable («ce qui devrait être») (Loubier 1994: 50).

Cette définition de la notion de besoin peut être ainsi représentée par ce diagramme:

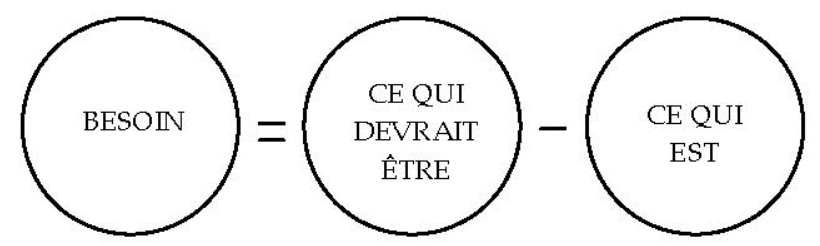


C'est sur base de nos motivations et en fonction des besoins à satisfaire que nous avions pu identifier: (a) le manque ou l'inexistence d'outils didactiques et lexicographiques finnois-lingala, (b) apprendre le finnois, afin de nous intégrer en Finlande; et (c) élaborer un dictionnaire finnois-lingala-français à même d'aider aussi bien nous-mêmes que d'autres immigrants se trouvant dans la même situation que nous (quête d'intégration par la connaissance de la langue du pays d'accueil).

Pour atteindre ces objectifs, il fallait que nous puissions nous doter de ressources et moyens financiers conséquents. Nous avions procédé de la manière qu'illustre ce diagramme:

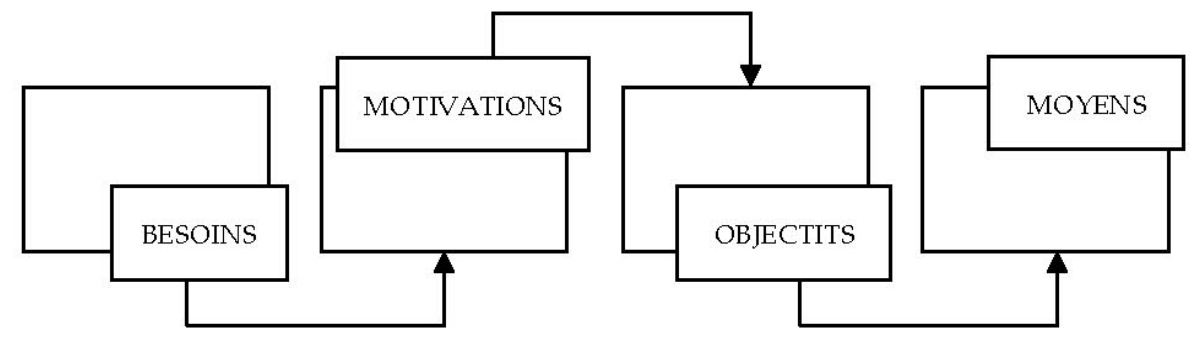

\subsection{Outil informatique de confection}

Par manque d'outil informatique approprié aux recherches dictionnairiques (les plus en vogue et les plus appropriés aux langues du domaine bantu étant les logiciels Shoebox/Toolbox ${ }^{2}$ de la Société Internationale de Linguistique et TshwaneLex, logiciels payants), nous nous étions résolu de travailler avec nos moyens de bord en ayant recours à un outil informatique d'un maniement facile, à savoir le logiciel de traitement des textes Word de la firme Microsoft.

\subsection{Le choix du lingala comme principale langue cible}

Pour bien comprendre les raisons ayant motivé le choix du lingala comme principale langue cible de ce dictionnaire, il convient de rappeler de manière succincte sa situation sociolinguistique au Congo-Kinshasa. En effet, le lingala est avec le swahili, l'haoussa et le wolof, une des langues supranationales que l'Afrique connaît aujourd'hui. Il fait partie de quatre langues nationales de la République Démocratique du Congo, les trois autres étant le kikongo, le kiswahili et le cilubà.

Langue de la capitale politique de la RD-Congo qui est Kinshasa, le lingala est en passe de devenir un super véhiculaire sur l'ensemble du territoire national rd-congolais, étant donné que beaucoup de Congolais qui émigrent à l'étranger et qui ne sont pas issus de la zone linguistique lingalophone, le 
comprennent et le parlent du fait qu'il est, entre autre, la langue de la musique congolaise moderne, la langue du théâtre national et la langue des affaires.

À l'étranger, c'est également le lingala qui, dans beaucoup de situations de communication, sert de langue d'intercommunication entre les membres de la diaspora congolaise issus d'aires linguistiques diverses. C'est ce qui justifie son choix comme principale langue cible de ce dictionnaire. Nous avons choisi le français comme langue

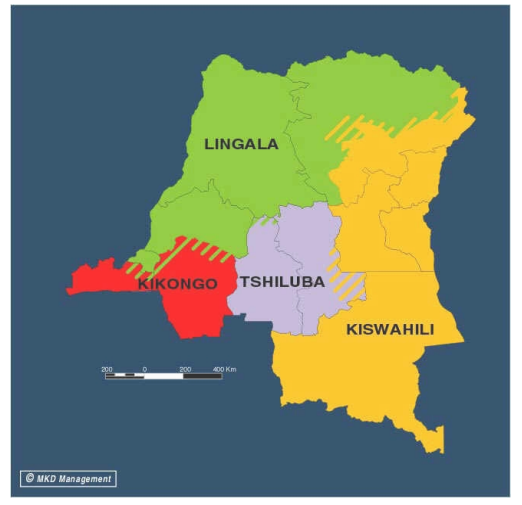
cible seconde afin qu'il serve également les congolais issus de trois autres aires linguistiques. Ce choix découle de son statut de langue officielle en RD-Congo.

\subsection{Fiche synoptique du dictionnaire}

\begin{tabular}{|l|l|}
\hline Intitulé du dictionnaire & Dictionnaire finnois-lingala-français \\
\hline Auteur & Kadima Batumona Adi Gilbert \\
\hline Statut & $\begin{array}{l}\text { Docteur ès lettres; Agrégé de l'enseignement secondaire } \\
\text { supérieur (option français) }\end{array}$ \\
\hline Support du dictionnaire & papier \\
\hline Pagination et format & 323 pages A4 \\
\hline Classement & alphabétique \\
\hline Public cible & (im)migrants lingalo-francophones établis en Finlande \\
\hline Fonctions & référence, apprentissage, décodage, enrichissement lexical \\
\hline Macrostructure & 123.754 mots; 18.450 entrées \\
\hline Microstructure & $\begin{array}{l}\text { équivalents en langues ciblées (lingala et français); origine } \\
\text { du mot si nécessaire; pas de marques d'usage, pas de pro- } \\
\text { nonciation }\end{array}$ \\
\hline Illustrations & 1 carte de 4 grandes aires linguistiques rd-congolaises \\
\hline
\end{tabular}

Ce dictionnaire a été élaboré aux fins d'aider de prime abord les congolais vivant en Finlande et servir à ces derniers d'outil d'appui dans l'apprentissage de la langue de leur pays d'accueil, le finnois. En tant qu'outil lexicographique, son rôle est de donner la maîtrise des moyens d'expression et d'accroître le savoir culturel de ses utilisateurs.

Avant sa validation comme dictionnaire pédagogique, il avait été soumis à l'approbation d'une commission chargée du contrôle des matériels pédagogiques de la Fondation Diakonia ${ }^{3}$. Cette commission comprenait un anthropologue, un professeur de langue finnoise (également francophone) et un spécialiste en sciences documentaires. L'approbation de notre dictionnaire par cette dernière commission tient entre autre au fait qu'il répond à ces exigences que le GRELEP pense comme celles, auxquelles doit devoir répondre tout diction- 
naire d'apprentissage: - tirer parti des dictionnaires existants - être basé sur un corpus - se fonder sur l'expérience didactique de professeurs - être accessible par le biais de la langue maternelle de l'apprenant et enfin, - constituer un outil d'(auto-)apprentissage, c'est-à-dire de construction du savoir.

\subsection{Extrait du dictionnaire}

\begin{tabular}{|c|c|}
\hline $\begin{array}{l}\text { concis } \\
\text { yö - butú - nuit } \\
\text { yöeläin - nyama ya botámboli na } \\
\text { butúbutú - animal nocturne } \\
\text { yöelämä - ezalela ya butú - vie } \\
\text { nocturne } \\
\text { yökerho - ekalá - boîte de nuit, boîte } \\
\text { yökyöpeli - motambolampímpa, } \\
\text { motámboli ya butú - couche-tard, } \\
\text { noctambule } \\
\text { yököttävä - ya bopési nkándá - } \\
\text { écoeurant, dégoûtant } \\
\text { yölento - mobémbo ya mpépo ya butú } \\
\text { - vol de nuit } \\
\text { yöllinen - ya butú - nocturne }\end{array}$ & $\begin{array}{l}\text { Zaïre - Zaire - Zaïre } \\
\text { zaïrelainen - zairwá, zét, ya mbóka } \\
\text { Zaire - zaïrois(e) } \\
\text { zeniitti - zeniti - zénith } \\
\text { zeppeliini - zepelé (< fr. zeppelin) - } \\
\text { zeppelin } \\
\text { Zimbabwe - Zimbábwe - Zimbabwe } \\
\text { zimbabwelainen - zimbabweyé - } \\
\text { zimbabwéen(ne) } \\
\text { zirkoni - zirkó (<fr. zircon) - zircon } \\
\text { zoomata - kozumé (< fr. zoomer) - } \\
\text { zoomer } \\
\text { zoomaus - zúm (< fr. zoom) - zoom } \\
\text { zoomojektivi - zúm - zoom }\end{array}$ \\
\hline
\end{tabular}

\subsection{Caractéristiques techniques, macro- et microstructures}

\subsubsection{Caractéristiques techniques}

Comme nos lecteurs peuvent bien s'en rendre compte en passant en revue le tableau synoptique ainsi que l'extrait de la microstructure ci-dessus, notre dictionnaire finnois-lingala-français est: interlinguistique, trilingue et monodirectionnel. En effet, il est interlinguistique trilingue parce qu'il porte sur trois issues de trois familles différentes: le finnois (famille finno-ougrienne), le lingala (langue africaine bantu) et le français (langue indo-européenne); quant à sa monodirectionnalité, elle réside en ce qu'il part de la langue source vers les langues cibles. C'est dire qu'à la différence d'autres dictionnaires multilingues (dont les bilingues surtout) qui sont bidirectionnels (se déployant de la source vers la cible et de la cible vers la source), et qui sont à la fois des dictionnaires de version et de thème, le nôtre est uniquement un dictionnaire de version étant donné qu'il se déploie dans un seul et unique sens.

Rey-Debove (2005) établit, pour sa part, la distinction suivante entre les dictionnaires monolingues et les multilingues:

Le dictionnaire multilingue réalise un trajet qui consiste en un décodage (traduction) et en un encodage (production): langue $\mathrm{A} \rightarrow$ langue $\mathrm{B} \rightarrow$ langue $\mathrm{C}$. Ce n'est que le passage d'un code lexical à un autre et est généralement dépourvu de définition. Le dictionnaire monolingue, quant à lui, est destiné à un éclaircissement d'une langue A pour un lecteur A. Il révèle seulement le sens (= traduction) mais jamais la production (= encodage). Bien plus, il tente de décrire la langue par le lexique, en produisant des exemples de signes intégrés dans une syntaxe 
avec leur morphosyntaxe. Ainsi donc, grâce à l'exemple on accède au sens du mot, à la grammaire, et à la sémiologie d'une société à un moment donné.

\subsubsection{Macro- et microstructures}

Du point de vue sa forme, notre dictionnaire finnois-lingala-français comme bon nombre de dictionnaires, comprend une macrostructure et une microstructure. La macrostructure est l'ensemble des adresses ou entrées tandis que la microstructure, elle, est l'ensemble des éléments informationnels accompagnant chacune des entrées d'un dictionnaire. Les détails relatifs à la macrostructure étant fournis dans la fiche synoptique ci-dessus, il ne nous reste qu'à apporter quelques éclaircissements quant à la manière dont nous avons organisé la microstructure du dictionnaire.

En effet, nous nous sommes référé à la tradition lexicographique qui est en vigueur en Finlande, où nous sommes établis et où le dictionnaire fut élaboré et destiné à y être utilisé. Dans ce pays et cela, en tenant compte de langues en présence, les microstructures d'un dictionnaire bilingue ou trilingue comme le nôtre s'articulent d'ordinaire autour des élements ci-après: prononciation, équivalents en langue(s) ciblée(s) et quelques marques d'usage (genre, nombre, etc.). Le lingala se lisant comme le finnois et ne faisant nullement pas de distinction quant au genre et au nombre, nous avons jugé bon de ne pas inscrire dans l'article ces marques d'usage ainsi que la prononciation. Ainsi donc, la microstructure de notre dictionnaire se limite à l'équivalent et dans certains cas s'étend à l'origine du mot s'il s'agit d'un emprunt. Dans l'éxtrait en question, les équivalents en lingala de ces trois entrées: zoomata, zoomaus et zirkoni sont accompagnés des marques relatives à leurs origines (ce sont des emprunts faits au français).

Voici à titre d'exemples, trois microstructures des dictionnaires bilingues en usage en Finlande et dont deux font partie de notre corpus. Pour des raisons d'économie, nous ne mentionnerons qu'une direction par dictionnaire.

\section{- Dictionnaire finnois-français-finnois de Kalmbach et Sundelin Finnois-français:}

\begin{tabular}{|c|c|}
\hline $\begin{array}{l}\text { aaltoilu ondoiement } m \text {, ondula- } \\
\text { tion } f \\
\text { aaltoliike mouvement } m \text { ondula- } \\
\text { toire; (kuv) va-et-vient } m \text { (taipum) } \\
\text { aaltomainen ondoyant, onduleux } \\
(m) \text {, onduleuse }(f) \\
\text { aaltopahvi carton } m \text { ondulé } \\
\text { aaltopelti tôle } f \text { ondulée } \\
\text { aaltopituus (kuv) samaila aalto- } \\
\text { pituudella sur la même longueur } \\
\text { d'onde }\end{array}$ & $\begin{array}{l}\text { Wä le matin, pendant la matinée, } \\
\text { avant midi } \\
\text { aamurusko aurore } f \\
\text { aamutak|ki robe } f \text { de chambre, } \\
\text { (kevyt naisen aamutakki myös) } \\
\text { peignoir m; -issa en robe de } \\
\text { chambre, en peignoir } \\
\text { aamutuimaan de bonne heure } \\
\text { aamu-uninen lève-tard mf (tai- } \\
\text { pum) } \\
\text { aamuvirkku matinal (mpl mati- }\end{array}$ \\
\hline
\end{tabular}




\begin{tabular}{|ll|}
\hline Zaïre $m$ Zaire & zénith $m$ huippu, zeniitti \\
zaïrois zairelainen & Z.E.P. (lyh. sanoista zone d'éducation \\
Zambie $f$ Sambia & prioritaire) (läh.) ongelmakoulualue \\
zambien, zambienne sambialainen & zéro nolla \\
zantedescia $f$ kalla & zeste $m 1$ pala sirushedelmän kuorta \\
zapper vaihtala koko ajan TV-kanavaa; & 2 (kuv.) pieni märärä \\
(kuv.) hyppiä asiasta toiseen & zézaiement $m$ ässävika \\
zapping $m$ kanavasurllailu & zézayer lespata \\
zébré juovikas & Z.l. (lyh. sanoista zone industrielle) teol- \\
zèbre $m$ seepra quel drôle de zèbre! & lisuusalue \\
(ark., kuv.) mikă hassu tyyppi! & zieuter (sl.) katsoa \\
zebrina $f$ (kasv.) juoru & zigouiller (sl.) tappaal \\
\hline
\end{tabular}

\section{- Dictionnaire finnois-allemand-finnois de Klemmt et al.} Allemand-finnois:

\begin{tabular}{|ll|}
\hline zack interj nopeasti & zählbar adj joka voidaan laskea \\
Zack auf Zack sein (ihminen) olla & zahlen v 1 maksaa er hat zuviel dafür \\
valmiina/kunnossa, osata asiansa; & bezahlt hän maksoi siitä liikaa eine \\
(asiat) olla reilassa & Rechnung zahlen (ark) maksaa lasku \\
Zacke die Zacke, die Zacken piikki, & 2 zahlen, bitte! saanko laskun? \\
(vuoren)huippu, (sahanterän) & zählen $v 1$ laskea die Gäste zählen \\
hammas, (kamman, haravan) pii(kki) & laskea vieraat, laskea paljonko \\
zackig adj 1 rosoinen, rosoreunainen, & vieraita on 2 sie zählt 50 Jahre hän \\
pykäläinen 2 reipas & on 50 -vuotias 3 sie zählt ihn nicht zu \\
zagen $v$ empiä, aristella, haparoida & ihren Freunden hän ei katso miestä \\
(kuv) & ystäväkseen, hän ei katso miehen \\
zaghaft adj empivä, aristeleva, & kuuluvan ystäväpiiriinsä 4 sie zähit \\
haparoiva & zu den bedeutendsten Schrift- \\
Zaghaftigkeit die epävarmuus, & stellerinnen dieses Jahrhunderts hän \\
aristelu & on yksi tämän vuosisadan \\
zäh adj 1 sitkeä, (neste) jähmeä & merkittävimpiä (nais)kirjailijoita \\
2 (kuv) sinnikäs, sitkeä, sisukas & $\mathbf{5}$ kann ich auf dich zählen? voinko \\
3 hidas & luottaa sinuun? voinko laskea sinun \\
zähflüssig adj jähmeä & varaan? \\
\hline
\end{tabular}

\subsubsection{Démarche lexicographique}

Nous basant sur ces deux caractéristiques susmentionnées (interlinguistique trilingue et monodirectionnel), nous avons organisé nos adresses de manière onomasiologique, c'est-à-dire que nos adresses ou entrées sont présentées suivant l'ordre: vedette - équivalent ou synonyme; en cas de difficulté de trouver un synonyme, nous avons fait recours soit à l'emprunt, à un calque ou encore à une explication de la vedette en langue cible.

Comme dans tout outil lexicographique multilingue, la recherche d'un équivalent pose le plus souvent des problèmes, dans la mesure où il arrive qu'une notion dans la langue source n'ait pas de correspondant dans la (les) 
langue(s) ciblée(s). Aux fins de faire face à cette difficulté, nous avons dû recourir aux techniques d'enrichissement qui sont d'usage courant aussi bien en lexicologie qu'en terminologie. Ces techniques sont résumées par Edema (2008: 7273) en termes suivants:

Pour s'enrichir, toute langue dispose de trois bases: une base grammaticale qui, avec ses structures, aide à former de nouveaux mots, une base sémantique qui, avec des mots déjà existants, donne de nouveaux sens; et l'emprunt à d'autres langues, source également de nouveaux mots pour une langue. Les deux premières bases sont qualifiées de bases internes tandis que la dernière, à savoir l'emprunt, elle, est qualifiée de base externe.

De l'avis de Adamska-Sałaciak (2011) qui réfère aux recherches menées par Zgusta (1971, 1987), les équivalences dans un dictionnaire bilingue ou multilingue peuvent être de différentes natures: cognitive, traductologique, explicative et fonctionnelle. À la question de savoir à quel type d'équivalences avonsnous eu recours de notre travail, nous répondrons qu'avant même que AdamskaSałaciak n'ait publié son étude, notre dictionnaire datant de 2007 et donc antérieur à cette étude, était déjà paru. Mais si nous essayons de scruter aujourd'hui les équivalences correspondant aux adresses en langue source, nous pouvons dire qu'elles sont de trois natures: cognitive, traductologique et explicative.

Exemples:

\section{- Équivalences cognitives}

\begin{tabular}{|l|l|l|}
\hline Finnois & Lingala & Français \\
\hline isä & tatá & père, papa \\
\hline äiti & mamá & mère, maman \\
\hline lapsi & mwána & enfant \\
\hline koti & ndáko & maison \\
\hline silmä & lísu & oil \\
\hline koulu & etéyelo & école \\
\hline aamu & ntóngó & matin \\
\hline ilta & mpókwa & soir \\
\hline
\end{tabular}

\section{- Équivalences traductologiques}

\begin{tabular}{|l|l|l|}
\hline Finnois & Lingala & Français \\
\hline omakotitalo & ndáko ya moto mókó & villa \\
\hline etunimi & nkómbó ya yambo & prénom \\
\hline öljyväri & lángi ya mafúta & couleur à huile \\
\hline yleistää & kosangisa esíká mókó & généraliser \\
\hline virusoppi & boyébi ya maye matálí mikróbi & virologie \\
\hline allekirjoittaa & kotiýa sinyé & signer \\
\hline
\end{tabular}


- Équivalences explicatives

\begin{tabular}{|l|l|l|}
\hline Finnois & Lingala & Français \\
\hline divari & wenze ya kowet; wenze ya bwáká-nzóto & brocante \\
\hline elokuu & sánzá ya mwambe & août \\
\hline hedelmätarha & elanga ya malálá & verger \\
\hline öljyvahinko & likámá eútí na bosopani ya pitoló & accident pétrolier \\
\hline huomena & lóbí óy'ezalí koyá & demain \\
\hline
\end{tabular}

\subsubsection{Grille de création lexicale}

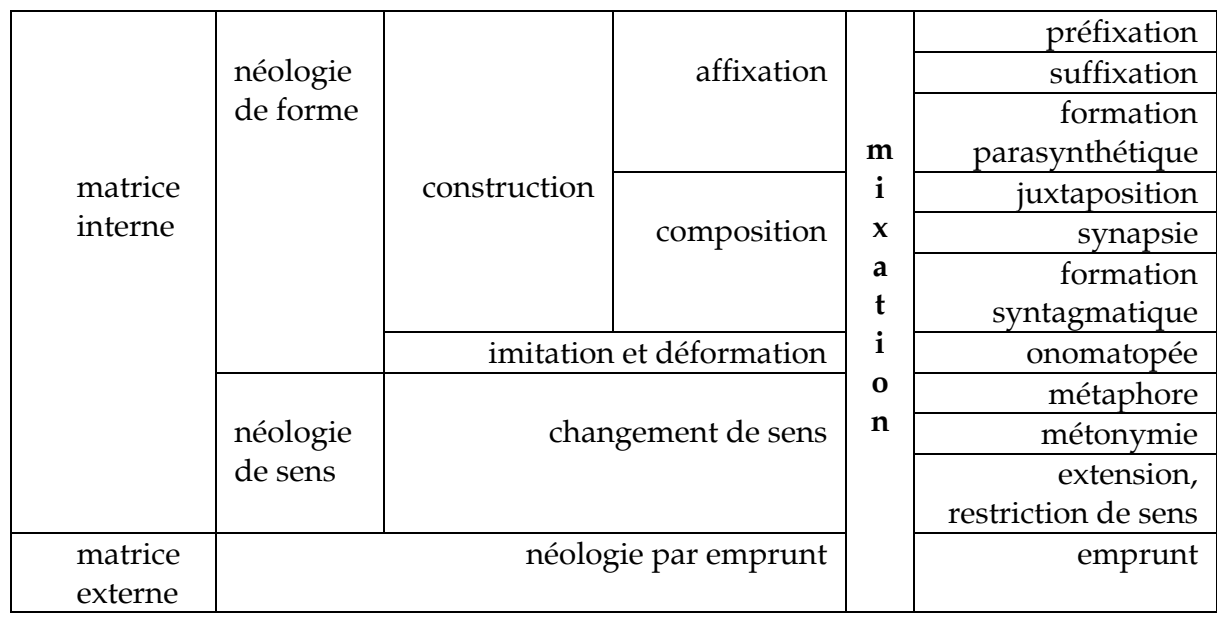

Cette grille nous a servi d'outil pour la formation des nouvelles lexies lingala et ce, aux fins de combler des vides lexicaux. Ces quelques exemples qui suivent montrent les matrices lexicales auxquelles nous avons eu recours dans nos opérations de création lexicale. Les matrices les plus sollicitées sont: l'emprunt, la composition par juxtaposition, la composition synaptique, la formation syntagmatique, la dérivation parasynthétique, la préfixation et la suffixation.

\subsubsection{Quelques exemples de création lexicale}

\section{- L'emprunt}

Exemples:

\begin{tabular}{|l|l|l|l|}
\hline Finnois & Lingala & Français & Origine \\
\hline hissi & asanséli & ascendeur & français \\
\hline juusto & fromázi & fromage & français \\
\hline lumi & nézi & neige & français \\
\hline
\end{tabular}




\begin{tabular}{|l|l|l|l|}
\hline sauna & saúna & sauna & finnois \\
\hline kaukosäädin & telekomande & télécommande & français \\
\hline
\end{tabular}

- La composition par juxtaposition

C'est un mode de composition dans lequel les éléments lexicaux de forme pleine avec ou sans trait d'union sont réunis en mot composé (Boutin-Quesnel 1990: 24).

Exemples:

\begin{tabular}{|l|l|l|l|l|}
\hline \multirow{2}{*}{ Finnois } & \multicolumn{3}{|c|}{ Lingala } & \multirow{2}{*}{ Français } \\
\cline { 2 - 4 } & déterminé & déterminant & lexie composée & budgétiser \\
\hline budjetoida & kosála & bidié & kosála bidié & déléguer \\
\hline delegoida & kotínda & ntómá & kotínda ntómá & décoder \\
\hline dekoodata & kolongola & kóde & kolongola kóde & dévaluer \\
\hline devalvoida & kokitisa & motúyá & kokitisa motúyá & dén film \\
\hline elokuvata & kokanga & bilili & kokanga bilili & tourner un \\
\hline
\end{tabular}

\section{- La composition synaptique}

C'est le mode de composition par lequel le déterminé est relié au déterminant par le truchement d'un mot de liaison couramment appelé connectif, joncteur ou déterminatif du point de vue fonctionnel.

Exemples:

\begin{tabular}{|c|c|c|c|c|}
\hline \multirow[b]{2}{*}{ Finnois } & \multicolumn{3}{|c|}{ Lingala } & \multirow[b]{2}{*}{ Français } \\
\hline & déterminé & déterminatif & déterminant & \\
\hline autoliikenne & botámboli & ya & mítuka & circulation automobile \\
\hline autojono & molongó & ya & mítuka & queue de voitures \\
\hline bussikaista & nzelá & ya & bísi & $\begin{array}{l}\text { couloir réservé aux } \\
\text { autobus }\end{array}$ \\
\hline croissant & lípa & ya & krwasa & croissant \\
\hline desinfiointiaine & nkísi & ya & kopétola & désinfectant \\
\hline
\end{tabular}

\section{- La formation syntagmatique}

Nous entendons par la formation syntagmatique un mode de composition qui conduit à la formation d'un syntagme lexical. Ce syntagme peut être construit sur le modèle de la composition synaptique, de la composition par juxtaposition ou une combinaison de ces deux formes de composition.

Exemples:

\begin{tabular}{|l|l|l|}
\hline Finnois & Lingala & Français \\
\hline Euroopan unioni & lisanga ya bambóka ya poto & Union européenne \\
\hline isällisesti & na ndengé ya botatá & paternellement \\
\hline
\end{tabular}




\begin{tabular}{|l|l|l|}
\hline desinfiointiaine & nkísi ya koboma mikróbi & désinfectant \\
\hline omakotitalo & ndáko ya moto mókó & villa \\
\hline ompelimo & ndáko ya botongi bilambá & atelier de couture \\
\hline
\end{tabular}

\section{- La dérivation parasynthétique}

Il y a formation parasynthétique lorsqu'un substantif dérivé réunit en lui les deux modes d'affixation, à savoir la préfixation et la suffixation.

Exemples:

\begin{tabular}{|l|l|l|l|l|l|l|}
\hline \multirow{2}{*}{ Finnois } & \multicolumn{5}{|c|}{ Lingala } & \multirow{2}{*}{} \\
\cline { 2 - 6 } & préfixe & radical & suffixe(s) & lexie dérivée & origine & Français \\
\hline organisointi & bo- & -bong- & -is-i & bobongisi & kobongisa & organisation \\
\hline painike & e- & -simb- & - -el-i & esímbelí & kosímba & levier, poignée \\
\hline pelokas & mo- & -báng- & - -i & mobángi & kobánga & peureux \\
\hline piirrin & n- & -lakis- & $-a$ & ndakisa & kolakisa & style \\
\hline ryhmitys & li- & -sang- & -a & lisangá & kosangisa & groupement \\
\hline
\end{tabular}

\section{- La préfixation}

Il y a donc préfixation lorsque l'affixation s'opère par l'adjonction des préfixes.

Exemples:

\begin{tabular}{|c|c|c|c|c|c|c|}
\hline \multirow[b]{2}{*}{ Finnois } & \multicolumn{5}{|c|}{ Lingala } & \multirow[b]{2}{*}{ Français } \\
\hline & préfixe & radical & suffixe(s) & lexie dérivée & origine & \\
\hline organisointi & bo- & -bong- & -is-i & bobongisi & kobongisa & organisation \\
\hline painike & e- & $-\operatorname{simb}-$ & -el-i & esímbelí & kosímba & levier, poignée \\
\hline pelokas & mo- & -báng- & $-\mathrm{i}$ & mobángi & kobánga & peureux \\
\hline piirrin & n- & -lakis- & $-a$ & ndakisa & kolakisa & style \\
\hline ryhmitys & li- & -sang- & $-a$ & lisangá & kosangisa & groupement \\
\hline
\end{tabular}

\section{- La suffixation}

On parle de la suffixation lorsque l'affixation s'opère par l'adjonction des suffixes.

Exemples:

\begin{tabular}{|l|l|l|l|l|l|l|}
\hline \multirow{2}{*}{ Finnois } & \multicolumn{5}{|c|}{ Lingala } & \multirow{2}{*}{} \\
\cline { 2 - 6 } & préfixe & radical & suffixe(s) & lexie dérivée & origine & Français \\
\hline organisointi & bo- & -bong- & -is-i & bobongisi & kobongisa & organisation \\
\hline painike & e- & -simb- & -el-i & esímbelí & kosímba & levier, poignée \\
\hline pelokas & mo- & -báng- & -i & mobángi & kobánga & peureux \\
\hline piirrin & n- & -lakis- & -a & ndakisa & kolakisa & style \\
\hline ryhmitys & li- & -sang- & -a & lisangá & kosangisa & groupement \\
\hline
\end{tabular}




\subsection{Difficulté rencontrée}

Le lingala, l'une des langues cibles de ce dictionnaire, comporte plusieurs variétés dialectales. En sus du problème lié au choix de la variété dialectale, nous avons eu aussi à faire face aux problèmes liés à l'orthographe. En effet, comme bon nombre des langues africaines «bantu», le lingala est une langue qui comporte des voyelles ouvertes et est une langue à tons.

Exemples:

- móto - tuli - feu

- moto - ihminen - être humain

- motó - pää - tête

Eu égard à la difficulté de nous procurer un logiciel approprié au niveau de la notation tant des tons que des voyelles ouvertes, nous avons estimé qu'il n'était pas du tout nécessaire de généraliser la notation des tons et des voyelles ouvertes, car, avons-nous estimé, étant donné que le dictionnaire traite des mots isolés d'une langue, tout lingalophone est à même de faire la distinction entre les homographes et les homophones selon le contexte.

\section{Conclusion}

Sous le titre de «lexicographie et enseignement de langues liées à l'immigration: note sur le contexte sociolinguistique de l'élaboration du dictionnaire finnois-lingala-français», nous nous étions proposé de retracer l'itinéraire méthodologique que nous avions suivi en vue la rédaction de notre outil lexicographique dont la présente contribution en constitue une description.

Dans les pages qui précèdent, nous nous sommes efforcé de montrer comment l'immigration en tant phénomène social a généré une recherche lexicographique qui s'est soldée par la dictionnairie dotant le lingala d'un ouvrage lexicographique trilingue.

Tandis que nous sommes en train de préparer la seconde édition dudit ouvrage lexicographique trilingue, nous avons estimé qu'il était nécessaire de recueillir des critiques quant à la méthodologie utilisée lors de la première édition. Nous sommes conscient que cette dernière est perfectible et qu'elle doit être enrichie de nouvelles entrées. Mais déjà, telle qu'elle se présente, si elle peut continuer à aider les immigrés congolais en phase d'apprentissage du finnois et ce, en vue de leur intégration en Finlande, nous trouverions en eux une raison de parachever dans le meilleur délai la seconde édition améliorée qui est en préparation.

\section{Notes}

1. Lire à ce propos les Propositions du Groupe des Intellectuels pour le Dialogue Interculturel Constitué à l'initiative de la Commission Européenne, dans «comment la multiplicité des 
langues pourrait consolider l'Europe»?, Bruxelles 2008. Disponible en ligne sur: http:// www.europa.eu/languages/fr/document/106/5.

2. Ce logiciel, dans sa conception automatique de dictionnaire, permet d'organiser en champs les données exportables et convertibles pour la suite en articles ordinaires de dictionnaire, sous forme de document Word.

3. La Fondation Diakonia, connue sous la dénomination de Diakonia Laitos est des plus présentes fondations qui sont visibles en Finlande dans le domaine de l'éducation-formation et qui se distinguent dans les oeuvres de charité. C'est elle qui avait financé la première édition du dictionnaire finnois-lingala-français.

\section{Bibliographie}

Adamska-Sałaciak, Arleta. 2011. Between designer drugs and afterburners: A Lexicographic-Semantic Study of Equivalence. Lexikos 21: 1-22.

Agbetse, Yao. s.d. Contribution à la réflexion sur la problématique de la réalisation effective des droits culturels dans le contexte de la migration internationale. Disponible en ligne sur: http://www. unifr.ch/iiedh/assets/files/DS/DS5-dc-travailleurs-migrants.pdf.

Boutin-Quesnel, Rachel et al. 1990. Vocabulaire systématique de la terminologie. Disponible en ligne sur: http://www.oqlf.goav.qc.ca/ressources/bibliotheque/dictionnaires/voc_systematique_ terminologie.pdf. Consulté le 12 novembre 2010.

Bouquin-Keller, Hélène. 2001. Les langues dites «d'immigration» dans les bibliothèques municipales françaises. Disponible en ligne sur: http://bbf.enssib.fr/. Consulté le 22 août 2013.

Cabré, Maria Teresa. 2000. Terminologie et linguistique: la théorie des portes. Terminologies Nouvelles 21: 10-15, juin. Terminologie et diversité culturelle.

Centre national de ressources textuelles et lexicales. Lexicographie: définition. Disponible en ligne sur: http://www.cnrtl.fr/lexicographie/lexicographie.

Conseil de l'Europe: Unité des politiques linguistiques. Cadre européen commun de référence por les langues: apprendre, enseigner, évaluer. Disponible en ligne sur: http://www.coe.int/lang-CECR.

Edema, Atibakwa Baboya. 1994. Dictionnaire bangala-français-lingala. Saint-Maur: Sépia-ACCT.

Edema, Atibakwa Baboya. 2008. La construction du signifiant. Didi-Kidiri, Marcel (Ed.). 2008. Le vocabulaire scientifique dans les langues africaines. Pour une approche culturelle de la terminologie: 71-99. Paris: Karthala.

Groupe de recherche en lexicographie pédagogique. s.d. Objectifs. Disponible en ligne sur: http://www.kuleuven.be/grelep/objectif/objectin.htm.

HCDH. 1985. La Déclaration sur les droits de l'homme des personnes qui ne possèdent pas la nationalité du pays dans lequel elles vivent. Adoptée le 13 décembre 1985 par consensus et contenue dans la résolution 40/144.

Helsingin Kaupunki-Opetusvirasto $=$ Education Department of City of Helsinki. s.d. Oman äidinkielen, kotikielen ja ylläpitokielen. opetus — opas kouluille. Disponible en ligne sur: http://www. kotisivukone.com/aidinkielenopetus.

Helsingin Tulkkikeskus Oy = Helsinki Interpretation Centre Ltd. Interpretation and Translations with I.A. Disponible en ligne sur: http://www.helsingintulkkikeskus.fi/en/languages.

Huck, Dominique. 2012. Avant-propos: Les langues des enfants «issus de l'immigration». Disponible en ligne sur: http://www.cahiersdugepe.fr. 
Hurme, Raija et al. 2007. Suomi-englanti-suomi sanakirja. 10ème édition. Helsinki: WSOY.

Kalmbach, Jean-Michel et Seppo Sundelin. 2008. Suomi-ranska-suomi sanakirja. 9ème édition. Helsinki: WSOY.

Kawata Ashem Tem, François Thomas. 2004. Bagó-Dictionnaire lingála-falansé/française-lingála. ParisSaint-Pierre-lès-Nemours: Karthala.

Klemmt, Rolf et al. 1992. Suomi-saksa-suomi sanakirja. Jyväskylä: Gummerus.

Lattunen, Hélène et Kari Viljanen. 2000. Dictionnaire finnois-français-finnois. 2ème édition. Helsinki: Gummerus.

Le Petit Larousse illustré. 2009. Paris: Larousse.

Loubier, Christiane. 1994. L'Aménagement linguistique au Québec: Enjeux et perspectives. Coll. Langues et Sociétés. Québec: Office de la langue française.

Ministère québécois de l'Éducation, du Loisir et du Sport. 2012-2013. Matériel didactique approuvé pour l'éducation préscolaire et l'enseignement primaire 2012-2013. Disponible en ligne sur: http://www3.mels.gouv.qc.ca/bamd/Doc/Liste_primaire_fr_ref.pdf.

Murray, James. s.d. Petite histoire des dictionnaires. Disponible en ligne sur: http://www.etudeslitteraires.com/histoire-dictionnaires.php.

ONU. 1984. Dispositions internationales pour la protection des droits de l'homme des non-ressortissants (Publication des Nations Unies, $\mathrm{N}^{\circ}$ de vente: F.80.XIV.2.).

Opetushallitus. s.d. Omana ädinkielenä opetut kielet ja opetukseen osallistuneiden määrät vuonna 2011. Disponible en ligne sur: http://www.oph.fi/download/142341_Omana_aidinkielena_opetetut_ kielet_ja_opetukseen_osallistuneiden_maarat_vuonna_2011-PDF.pdf.

Quentin, Francine. 2002. L'héritage linguistique des immigrés en danger. Archives-RFI (Langues). Disponible en ligne sur: http://www.rfi.fr/actufr/articles/026/article_13364.asp.

Rey-Debove, Josette. 2005. Typologie des dictionnaires généraux monolingues de la langue actuelle. Disponible en ligne sur: http://amsacta.unibo.it/2301/i/Debove.pdf.

Romppanen, Birgitta (2001), citée dans Kotikielenseura. 2002. Uutta sanakirjatutkimusta. Disponible en ligne sur: http://www.kotikielenseura.fi/virittaja/hakemistot/jutut/2002_300.pdf.

Sisäasiainministeriö. 2011. Maahanmuuton vuosikatsaus. Disponible en ligne sur: http://www.migri. fi/download/34214_maahanmuutto_2011_tilastokatsaus.pdf.

Unesco. 2003. La promotion des langues des communautes issues de l'immigration en débat. Disponible en ligne sur: http://www.unesco.org/most/discuss.htm.

Väestörekisterikeskus. 2011. Väestörakenne 2010. Dsponible en ligne sur: http://www.stat.fi/til/ vaerak/2010/vaerak_2010_2011-03-18_fi.pdf.

Zgusta, L. 1971. Manual of Lexicography. La Haye: Mouton.

Zgusta, L. 1987. Translational Equivalence in a Bilingual Dictionary: Bahukosyam. Dictionaries: Journal of the Dictionary Society of North America 9: 1-47. 